\title{
On Fourth-Order Elliptic Equations of Kirchhoff Type with Dependence on the Gradient and the Laplacian
}

\author{
Yuanfang $R u,{ }^{1,2}$ Fanglei Wang $\mathbb{D}^{1},{ }^{1}$ Yunhai Wang $\mathbb{D},{ }^{3}$ and Tianqing $A n^{1}$ \\ ${ }^{1}$ College of Science, Hohai University, Nanjing 210098, China \\ ${ }^{2}$ College of Science, China Pharmaceutical University, Nanjing 211198, China \\ ${ }^{3}$ School of Mechanical Engineering, Guizhou Institute of Technology, Guiyang 550003, China
}

Correspondence should be addressed to Yunhai Wang; wangyhnuaa@126.com

Received 26 September 2017; Accepted 20 November 2017; Published 2 January 2018

Academic Editor: Dhananjay Gopal

Copyright (C) 2018 Yuanfang Ru et al. This is an open access article distributed under the Creative Commons Attribution License, which permits unrestricted use, distribution, and reproduction in any medium, provided the original work is properly cited.

We consider a nonlocal fourth-order elliptic equation of Kirchhoff type with dependence on the gradient and Laplacian $\Delta^{2} u-(a+$ $\left.b \int_{\Omega}|\nabla u|^{2} d x\right) \Delta u=f(x, u, \nabla u, \Delta u)$, in $\Omega, u=0, \Delta u=0$, on $\partial \Omega$, where $a, b$ are positive constants. We will show that there exists $b^{*}>0$ such that the problem has a nontrivial solution for $0<b<b^{*}$ through an iterative method based on the mountain pass lemma and truncation method developed by De Figueiredo et al., 2004.

\section{Introduction}

This paper concerns with the existence of solutions of the fourth-order Kirchhoff type elliptic equation as follows:

$$
\begin{aligned}
\Delta^{2} u-\left(a+b \int_{\Omega}|\nabla u|^{2} d x\right) \Delta u & =f(x, u, \nabla u, \Delta u), \\
u & =0, \quad \text { in } \Omega, \\
\Delta u & =0,
\end{aligned}
$$

on $\partial \Omega$,

where $\Omega$ is a bounded and smooth domain in $R^{N}(N \geq 5), a$, $b$ are positive constants, and $f: \Omega \times R \times R^{N} \times R \rightarrow R$ is locally Lipschitz continuous.

The fourth-order elliptic equation

$$
\begin{aligned}
\Delta^{2} u-a \Delta u & =f(x, u), \quad \text { in } \Omega, \\
u & =0, \\
\Delta u & =0,
\end{aligned}
$$

arises in the study of traveling waves in suspension bridges, which has been extensively investigated in recent years, such as [1-6]. To our attention, some authors paid more attention to a more general biharmonic elliptic problem

$$
\begin{aligned}
\Delta^{2} u+q \Delta u+\alpha(x) u & =f(x, u, \Delta u, \nabla u), \quad \text { in } \Omega, \\
u(x) & =0, \\
\Delta u(x) & =0,
\end{aligned}
$$

on $\partial \Omega$.

For this problem, due to the presence of $\Delta u$ and $\nabla u$ in $f$, it is not variational. To overcome this difficulty, in [5], Wang deals with this problem via the upper and lower solutions and monotone iterative methods; in [7], the authors apply a technique developed by De Figueiredo et al. $[8,9]$ in studying a second-order elliptic problem involving the gradient, which "freezes" the gradient, and use truncation on the nonlinearity $f$. Thus the new problem becomes variational and an iterative scheme of the mountain pass "approximated" solutions is built.

In addition, the nonlocal fourth-order equation

$$
\Delta^{2} u-M\left(\int_{\Omega}|\nabla u|^{2} d x\right) \Delta u=f(x, u), \quad \text { in } \Omega,
$$

$$
u=0 \text {, }
$$




$$
\Delta u=0
$$

on $\partial \Omega$

has also been studied by many authors. We refer the readers to [10-20]. Particularly, Wang et al. studied the following fourthorder equation of Kirchhoff type equation

$$
\begin{aligned}
\Delta^{2} u-\lambda\left(a+b \int_{\Omega}|\nabla u|^{2} d x\right) \Delta u & =f(x, u), \quad \text { in } \Omega, \\
u & =0 \\
\Delta u & =0
\end{aligned}
$$

on $\partial \Omega$,

where $\lambda$ is a positive parameter. The authors showed that there exists $\lambda^{*}$ such that the fourth-order elliptic equation has a nontrivial solution for $0<\lambda<\lambda^{*}$ by using the mountain pass iterative techniques and the truncation method.

Motivated by these works, to study problem (1), we combine the famous mountain pass lemma with a technique developed by De Figueiredo et al. [8], which "freezes" the gradient and the Laplacian variable and use truncation on the nonlinearity of $f$. For convenience, we recall a definition and restate the mountain pass theorem.

Definition 1. Let $X$ be a real Banach space and $I: X \rightarrow R$ a $C^{1}$-functional. A sequence $\left\{u_{n}\right\}$ in $E$ is a (PS)-sequence for $I$ if $I\left(u_{n}\right) \rightarrow C$ for some constant $C \geq 0$ as $n \rightarrow \infty$, while $\left\langle I^{\prime}\left(u_{n}\right), u_{n}\right\rangle \rightarrow 0$ as $n \rightarrow \infty$. We say that the functional $I$ satisfies the (PS)-condition if any (PS)-sequence for $I$ has a convergent subsequence.

Theorem A (mountain pass lemma). Let $E$ be a real Banach space; $I \in C^{1}(E, R)$ satisfying (PS)-condition. Suppose the following:

(1) There exist $\rho>0, \alpha>0$ such that

$$
\left.I\right|_{\partial B_{\rho}} \geq I(0)+\alpha
$$

where $B_{\rho}=\{u \in E \mid\|u\| \leq \rho\}$.

(2) There is e $\in E$ and $\|e\|>\rho$ such that

$$
I(e) \leq I(0) \text {. }
$$

Then $I(u)$ has a critical value $c$ which can be characterized as

$$
C=\inf _{\gamma \in \Gamma} \max _{u \in \gamma([0,1])} I(u)
$$

where $\Gamma=\{\gamma \in C([0,1], E) \mid \gamma(0)=0, \gamma(1)=e\}$.

\section{The Main Result}

Theorem 2. Assume that the function $f$ satisfies the following conditions: $\left(f_{1}\right) f: \Omega \times R \times R^{N} \times R \rightarrow R$ is locally Lipschitz continuous, and there exist $d_{1}>0,1<p<(N+4) /(N-4)$ which satisfy $r:=r_{1}+r_{2}<2$ such that

$$
\left|f\left(x, t, \xi_{1}, \xi_{2}\right)\right| \leq d_{1}\left(1+|t|^{p}\right)\left(1+\left|\xi_{1}\right|\right)^{r_{1}}\left(1+\left|\xi_{2}\right|\right)^{r_{2}}
$$

for all $\left(x, t, \xi_{1}, \xi_{2}\right) \in \Omega \times R^{N+2}$.

$\left(f_{2}\right) \lim _{t \rightarrow 0}\left(f\left(x, t, \xi_{1}, \xi_{2}\right) / t\right)=0$ uniformly with respect to $x \in \Omega, \xi_{1} \in R^{N}$ and $\xi_{2} \in R$.

$\left(f_{3}\right)$ There exist $\Theta>2$ and $t_{1}>0$ such that

$$
\begin{aligned}
& 0<\Theta F\left(x, t, \xi_{1}, \xi_{2}\right) \leq t f\left(x, t, \xi_{1}, \xi_{2}\right), \\
& \forall|t| \geq t_{1}, x \in \Omega,\left(\xi_{1}, \xi_{2}\right) \in R^{N+1},
\end{aligned}
$$

where $F\left(x, t, \xi_{1}, \xi_{2}\right)=\int_{0}^{t} f\left(x, s, \xi_{1}, \xi_{2}\right) d s$.

$\left(f_{4}\right)$ There exist positive constants $\rho_{i}>0(i=1,2,3)$ depending on $a, b, \Theta$, and $d_{1}$ such that $L_{\rho_{i}}(i=1,2,3)$ satisfy

$$
\left(\tau_{1}^{2} L_{\rho_{1}}+\tau_{1} \tau_{2} L_{\rho_{2}}+\tau_{1} \tau_{3} L_{\rho_{3}}\right)<\min \{1, a\},
$$

where

$$
\begin{gathered}
L_{\rho_{1}}=\sup \left\{\frac{\left|f\left(x, t^{\prime}, \xi_{1}, \xi_{2}\right)-f\left(x, t^{\prime \prime}, \xi_{1}, \xi_{2}\right)\right|}{\left|t^{\prime}-t^{\prime \prime}\right|}: x\right. \\
\left.\in \Omega,\left|t^{\prime}\right|,\left|t^{\prime \prime}\right| \leq \rho_{1},\left|\xi_{1}\right| \leq \rho_{2},\left|\xi_{2}\right| \leq \rho_{3}\right\}, \\
L_{\rho_{2}}=\sup \left\{\frac{\left|f\left(x, t, \xi_{1}^{\prime}, \xi_{2}\right)-f\left(x, t, \xi_{1}^{\prime \prime}, \xi_{2}\right)\right|}{\left|\xi_{1}^{\prime}-\xi_{1}^{\prime \prime}\right|}: x\right. \\
\left.\in \Omega,|t| \leq \rho_{1},\left|\xi_{1}^{\prime}\right|,\left|\xi_{1}^{\prime \prime}\right| \leq \rho_{2},\left|\xi_{2}\right| \leq \rho_{3}\right\}, \\
L_{\rho_{3}}=\sup \left\{\frac{\left|f\left(x, t, \xi_{1}, \xi_{2}^{\prime}\right)-f\left(x, t, \xi_{1}, \xi_{2}^{\prime \prime}\right)\right|}{\left|\xi_{2}^{\prime}-\xi_{2}^{\prime \prime}\right|}: x\right. \\
\left.\quad \in \quad,|t| \leq \rho_{1},\left|\xi_{1}\right| \leq \rho_{2},\left|\xi_{2}^{\prime}\right|,\left|\xi_{2}^{\prime \prime}\right| \leq \rho_{3}\right\},
\end{gathered}
$$

and $\tau_{i}(i=1,2,3)$ are the optimal constants of the following inequalities:

$$
\begin{gathered}
\left(\int_{\Omega}|u|^{2} d x\right)^{1 / 2} \leq \tau_{1}\|u\|, \\
\left(\int_{\Omega}|\nabla u|^{2} d x\right)^{1 / 2} \leq \tau_{2}\|u\|, \\
\left(\int_{\Omega}|\Delta u|^{2} d x\right)^{1 / 2} \leq \tau_{3}\|u\|,
\end{gathered}
$$

where $\|\cdot\|$ is the norm of the Hilbert space $\mathbf{X}=\mathbf{H}^{2}(\Omega) \cap \mathbf{H}_{0}^{1}(\Omega)$ defined by

$$
\|u\|^{2}=\int_{\Omega}|\Delta u|^{2} d x+\int_{\Omega}|\nabla u|^{2} d x .
$$

Then there exists $b^{*}>0$ such that (1) has at least a nontrivial solution for $0<b<b^{*}$. 
For each $\omega \in \mathbf{X}$ and $\mathbf{R}>0$, we study the following "truncate" and "freezed" problem

$$
\begin{aligned}
\Delta^{2} u_{\omega}^{\mathbf{R}}-\Phi_{\mathbf{R}}(\nabla \omega) \Delta u_{\omega}^{\mathbf{R}} & =f_{\mathbf{R}}\left(x, u_{\omega}^{\mathbf{R}}, \nabla \omega, \Delta \omega\right), \quad \text { in } \Omega, \\
u_{\omega}^{\mathbf{R}} & =0 \\
\Delta u_{\omega}^{\mathbf{R}} & =0
\end{aligned}
$$

on $\partial \Omega$,

where

$$
\begin{aligned}
f_{\mathbf{R}}(x, t, \nabla \omega, \Delta \omega) & =f\left(x, t, \nabla \omega \varphi_{\mathbf{R}}(\nabla \omega), \Delta \omega \varphi_{\mathbf{R}}(\Delta \omega)\right), \\
\Phi_{\mathbf{R}}(\nabla \omega) & =a+b \int_{\Omega}\left|\nabla \omega \varphi_{\mathbf{R}}(\nabla \omega)\right|^{2} d x,
\end{aligned}
$$

$\varphi_{\mathbf{R}} \in C^{1}(R, R)$ satisfies $\left|\varphi_{\mathbf{R}}\right| \leq 1$, and

$$
\varphi_{\mathbf{R}}(\xi)= \begin{cases}1, & \text { if }|\xi| \leq \mathbf{R} \\ 0, & \text { if }|\xi| \geq \mathbf{R}+1\end{cases}
$$

The associated functional $J_{\omega}^{\mathbf{R}}: \mathbf{X} \rightarrow R$ is defined as

$$
\begin{aligned}
J_{\omega}^{\mathbf{R}}\left(u_{\omega}^{\mathbf{R}}\right)= & \frac{1}{2} \int_{\Omega}\left|\Delta u_{\omega}^{\mathbf{R}}\right|^{2} d x+\frac{1}{2} \Phi_{\mathbf{R}}(\nabla \omega) \int_{\Omega}\left|\nabla u_{\omega}^{\mathbf{R}}\right|^{2} d x \\
& -\int_{\Omega} F_{\mathbf{R}}\left(x, u_{\omega}^{\mathbf{R}}, \nabla \omega, \Delta \omega\right) d x,
\end{aligned}
$$

where

$$
F_{\mathbf{R}}(x, t, \nabla \omega, \Delta \omega)=\int_{0}^{t} f_{\mathbf{R}}(x, s, \nabla \omega, \Delta \omega) d s .
$$

Lemma 3. Let $\mathbf{R}>0$ and $\omega \in \mathbf{X}$ be fixed. Then

(1) there exist constants $\rho>0, \alpha>0$ such that $\left.J_{\omega}^{\mathbf{R}}(v)\right|_{\partial B_{\rho}} \geq$ $\alpha$ with $B_{\rho}=\{v \in \mathbf{X}:\|v\| \leq \rho\} ;$

(2) for fixed $v$ with $\|v\|=1, J_{\omega}^{\mathbf{R}}(t v) \rightarrow-\infty$ as $t \rightarrow+\infty$.

Proof. On one hand, by $\left(f_{2}\right)$, for any $\varepsilon>0$, there exists a constant $\sigma>0$ such that, for $|t|<\sigma$, one has

$$
F_{\mathbf{R}}(x, t, \nabla \omega, \Delta \omega) \leq \frac{1}{2} \varepsilon t^{2} .
$$

On the other hand, if $|t|>\sigma$, from $\left(f_{1}\right)$ it follows that there exists $C_{1}>0$ such that

$$
F_{\mathbf{R}}(x, t, \nabla \omega, \Delta \omega) \leq C_{1}|t|^{p+1}(\mathbf{R}+2)^{r} .
$$

Then, from (20), (21), and the Sobolev inequality, we have

$$
\begin{aligned}
J_{\omega}^{\mathbf{R}}(v)= & \frac{1}{2} \int_{\Omega}|\Delta v|^{2} d x+\frac{1}{2} \Phi_{\mathbf{R}}(\nabla \omega) \int_{\Omega}|\nabla v|^{2} d x \\
& -\int_{\Omega} F_{\mathbf{R}}(x, v \nabla \omega, \Delta \omega) d x \\
\geq & \frac{1}{2} \int_{\Omega}|\Delta v|^{2} d x+\frac{1}{2} \Phi_{\mathbf{R}}(\nabla \omega) \int_{\Omega}|\nabla v|^{2} d x \\
& -\frac{\epsilon}{2} \int_{\Omega}|v|^{2} d x-C_{1}(\mathbf{R}+2)^{r} \int_{\Omega}|v|^{p+1} d x \\
\geq & \left.\frac{\min \{1, a\}}{2}-\frac{\epsilon C}{2}\right)\|v\|^{2}-C(\mathbf{R}+2)^{r}\|v\|^{p+1}
\end{aligned}
$$

for some positive constant $C$. Therefore, for sufficiently small $\epsilon>0$, we can choose $\rho>0$ and $\alpha>0$ such that the first result of Lemma 3 holds.

Now, we show that $\left(f_{3}\right)$ implies that there exist $a_{2}, a_{3}>0$ such that

$$
\begin{aligned}
& F_{\mathbf{R}}\left(x, t, \xi_{1}, \xi_{2}\right) \geq a_{2}|t|^{\theta}-a_{3}, \\
& \forall x \in \Omega, t \in R, \xi_{1} \in R^{N}, \xi_{2} \in R .
\end{aligned}
$$

In fact, from $\left(f_{3}\right)$, we have $f\left(x, t, \xi_{1}, \xi_{2}\right) / F\left(x, t, \xi_{1}, \xi_{2}\right) \geq \theta / t$, for any $|t| \geq t_{1}$. Being integral from $t_{1}$ to $t$, we get

$$
\ln F\left(x, t, \xi_{1}, \xi_{2}\right)-\ln F\left(x, t_{1}, \xi_{1}, \xi_{2}\right) \geq \theta\left(\ln t-\ln t_{1}\right) ;
$$

namely,

$$
F\left(x, t, \xi_{1}, \xi_{2}\right) \geq \frac{F\left(x, t_{1}, \xi_{1}, \xi_{2}\right)}{t_{1}^{\theta}}|t|^{\theta}, \quad \forall|t| \geq t_{1} .
$$

Then

$$
\begin{aligned}
F_{\mathbf{R}}\left(x, t, \xi_{1}, \xi_{2}\right) & =F\left(x, t, \xi_{1} \varphi_{\mathbf{R}}\left(\xi_{1}\right), \xi_{2} \varphi_{\mathbf{R}}\left(\xi_{2}\right)\right) \\
& \geq \frac{|t|^{\theta}}{t_{1}^{\theta}} F\left(x, t_{1}, \xi_{1} \varphi_{\mathbf{R}}\left(\xi_{1}\right), \xi_{2} \varphi_{\mathbf{R}}\left(\xi_{2}\right)\right) .
\end{aligned}
$$

Let

$$
\begin{aligned}
a_{2} & =\frac{1}{t_{1}^{\theta}} \min _{x \in \Omega, \xi_{1} \in R^{N}, \xi_{2} \in R} F\left(x, t_{1}, \xi_{1} \varphi_{\mathbf{R}}\left(\xi_{1}\right), \xi_{2} \varphi_{\mathbf{R}}\left(\xi_{2}\right)\right) \\
& =\frac{1}{t_{1}^{\theta}} \min _{x \in \Omega,\left|\xi_{1}\right| \leq \mathbf{R}+1,\left|\xi_{2}\right| \leq \mathbf{R}+1} F\left(x, t_{1}, \xi_{1}, \xi_{2}\right)>0, \\
a_{3} & =\max _{x \in \Omega,|t| \leq t_{1},\left|\xi_{1}\right| \leq \mathbf{R}+1,\left|\xi_{2}\right| \leq \mathbf{R}+1} F\left(x, t_{1}, \xi_{1}, \xi_{2}\right)>0,
\end{aligned}
$$

and then inequality (23) holds.

Taking an arbitrary $v \in \mathbf{X}$ with $\|v\|=1$, then from (23), we get

$$
\begin{aligned}
J_{\omega}^{\mathbf{R}}(t v)= & \frac{1}{2} \int_{\Omega}|\Delta t v|^{2} d x+\frac{1}{2} \Phi_{\mathbf{R}}(\nabla \omega) \int_{\Omega}|\nabla t v|^{2} d x \\
& -\int_{\Omega} F_{\mathbf{R}}(x, t v, \nabla \omega, \Delta \omega) d x \\
\leq & \frac{1}{2} \int_{\Omega}|\Delta t v|^{2} d x+\frac{1}{2} \Phi_{\mathbf{R}}(\nabla v) \int_{\Omega}|\nabla t v|^{2} d x \\
& -a_{2} t^{\Theta} \int_{\Omega}|v|^{\Theta} d x+a_{3}|\Omega| \\
\leq & \frac{\max _{2}\left\{1, \Phi_{\mathbf{R}}(\nabla \omega)\right\}}{2} t^{2}\|v\|^{2}-a_{2} t^{\Theta} \int_{\Omega}|v|^{\Theta} d x \\
& +a_{3}|\Omega| \longrightarrow-\infty, \quad \text { as } t \longrightarrow+\infty,
\end{aligned}
$$

which implies that the second result of Lemma 3 holds.

Lemma 4. Let $\mathbf{R}>0$ and $\omega \in \mathbf{X}$ be fixed. Then the functional $J_{\omega}^{\mathbf{R}}(\cdot)$ satisfies the (PS)-condition. 
Proof. Let $\left\{u_{n}\right\} \subset \mathbf{X}$ be a (PS)-sequence; namely,

$$
\begin{aligned}
J_{\omega}^{\mathbf{R}}\left(u_{n}\right) & \longrightarrow C, \\
\left\langle J_{\omega}^{\prime \mathbf{R}}\left(u_{n}\right), u_{n}\right\rangle & \longrightarrow 0
\end{aligned}
$$

as $n \longrightarrow \infty$.

From the standard processes, we only need to prove that $\left\{u_{n}\right\}$ is bounded in $\mathbf{X}$. On a contradiction, suppose that $\left\|u_{n}\right\| \rightarrow$ $+\infty$; then, from $\left(f_{3}\right)$, we obtain

$$
\begin{aligned}
& J_{\omega}^{\mathbf{R}}\left(u_{n}\right)-\frac{1}{\Theta}\left\langle J_{\omega}^{\prime \mathbf{R}}\left(u_{n}\right), u_{n}\right\rangle=\frac{1}{2} \int_{\Omega}\left|\Delta u_{n}\right|^{2} d x+\frac{1}{2} \\
& \cdot \Phi_{\mathbf{R}}(\nabla \omega) \int_{\Omega}\left|\nabla u_{n}\right|^{2} d x \\
& -\int_{\Omega} F_{\mathbf{R}}\left(x, u_{n}, \nabla \omega, \Delta \omega\right) d x-\frac{1}{\Theta} \int_{\Omega}\left|\Delta u_{n}\right|^{2} d x \\
& -\frac{1}{\Theta} \Phi_{\mathbf{R}}(\nabla \omega) \int_{\Omega}\left|\nabla u_{n}\right|^{2} d x+\frac{1}{\Theta} \\
& \cdot \int_{\Omega} f_{\mathbf{R}}\left(x, u_{n}, \nabla \omega, \Delta \omega\right) u_{n} d x=\left(\frac{1}{2}-\frac{1}{\Theta}\right) \\
& \cdot\left[\int_{\Omega}\left|\Delta u_{n}\right|^{2} d x+\Phi_{\mathbf{R}}(\nabla \omega) \int_{\Omega}\left|\nabla u_{n}\right|^{2} d x\right] \\
& +\int_{\Omega}\left[\frac{1}{\Theta} f_{\mathbf{R}}\left(x, u_{n}, \nabla \omega, \Delta \omega\right) u_{n}\right. \\
& \left.-F_{\mathbf{R}}\left(x, u_{n}, \nabla \omega, \Delta \omega\right)\right] d x \geq\left(\frac{1}{2}-\frac{1}{\Theta}\right) \\
& \cdot \min \{1, a\}\|u\|^{2}+\int_{\Omega}\left[\frac{1}{\Theta} f_{\mathbf{R}}\left(x, u_{n}, \nabla \omega, \Delta \omega\right) u_{n}\right. \\
& \left.-F_{\mathbf{R}}\left(x, u_{n}, \nabla \omega, \Delta \omega\right)\right] d x \geq\left(\frac{1}{2}-\frac{1}{\Theta}\right) \\
& \cdot \min \{1, a\}\|u\|^{2} .
\end{aligned}
$$

On the other hand, from (29) we know that

$$
J_{\omega}^{\mathbf{R}}\left(u_{n}\right)-\frac{1}{\Theta}\left\langle J_{\omega}^{\prime \mathbf{R}}\left(u_{n}\right), u_{n}\right\rangle \leq C+C\left\|u_{n}\right\| .
$$

Then, from the above inequalities, we get

$$
\left(\frac{1}{2}-\frac{1}{\theta}\right) \min \{1, a\}\left\|u_{n}\right\|^{2} \leq C+C\left\|u_{n}\right\|,
$$

which contradicts with $\left\|u_{n}\right\| \rightarrow+\infty$. Therefore the sequence $\left\{u_{n}\right\}$ is bounded in $\mathbf{X}$.

Lemma 5. For any $\mathbf{R}>0$ and $\omega \in \mathbf{X}$, problem (15) has $a$ nontrivial weak solution.

Proof. By Theorem A, Lemmas 3, and 4, the result holds.

Lemma 6. Let $\mathbf{R}>0$ be fixed. Then there exist positive constants $\nu_{1}$ and $v_{2}:=v_{2}(\mathbf{R})$, independent of $\omega$, such that

$$
v_{1} \leq\left\|u_{\omega}^{\mathbf{R}}\right\| \leq v_{2}
$$

for every solution $u_{\omega}^{\mathbf{R}}$ obtained in Lemma 5 .
Proof. Firstly, since $J_{\omega}^{\mathbf{R}}\left(u_{\omega}^{\mathbf{R}}\right) \leq \max _{t \geq 0} J_{\omega}^{\mathbf{R}}(t v)$, from (23) it follows that

$$
\begin{aligned}
J_{\omega}^{\mathbf{R}}(t v) \leq & \frac{\max \left\{1, \Phi_{\mathbf{R}}\right\}}{2} t^{2}\|v\|^{2}-a_{2} t^{\Theta} \int_{\Omega}|v|^{\Theta} d x \\
& +a_{3}|\Omega| \\
\leq & \frac{\max \left\{1,[a+b(\mathbf{R}+1)]^{2}|\Omega|\right\}}{2} t^{2} \\
& -a_{2} t^{\Theta} \int_{\Omega}|v|^{\Theta} d x+a_{3}|\Omega| .
\end{aligned}
$$

As $\Theta>2$, we can get a $C>0$ such that $J_{\omega}^{\mathbf{R}}\left(u_{\omega}^{\mathbf{R}}\right) \leq C$; that is,

$$
\begin{gathered}
\frac{1}{2} \int_{\Omega}\left|\Delta u_{\omega}^{\mathbf{R}}\right|^{2} d x+\frac{1}{2} \Phi_{\mathbf{R}}(\nabla \omega) \int_{\Omega}\left|\nabla u_{\omega}^{\mathbf{R}}\right|^{2} d x \\
\leq C+\int_{\Omega} F_{\mathbf{R}}\left(x, u_{\omega}^{\mathbf{R}}, \nabla \omega, \Delta \omega\right) d x .
\end{gathered}
$$

Define $D=\left\{x \in \Omega:\left|u_{\omega}^{\mathbf{R}}\right|>t_{1}\right\}$, where $t_{1}$ is defined in $\left(f_{3}\right)$. Then we get

$$
\begin{aligned}
\frac{1}{2} \int_{\Omega}\left|\Delta u_{\omega}^{\mathbf{R}}\right|^{2} d x+\frac{1}{2} \Phi_{\mathbf{R}}(\nabla \omega) \int_{\Omega}\left|\nabla u_{\omega}^{\mathbf{R}}\right|^{2} d x \\
\leq C+\int_{\Omega} F_{\mathbf{R}}\left(x, u_{\omega}^{\mathbf{R}}, \nabla \omega, \Delta \omega\right) d x \\
=C+\int_{\Omega \backslash D} F_{\mathbf{R}}\left(x, u_{\omega}^{\mathbf{R}}, \nabla \omega, \Delta \omega\right) d x \\
\quad+\int_{D} F_{\mathbf{R}}\left(x, u_{\omega}^{\mathbf{R}}, \nabla \omega, \Delta \omega\right) d x \\
\leq C+a_{1}\left(t_{1}+\frac{\left|t_{1}\right|^{p+1}}{p+1}\right)(\mathbf{R}+2)^{r}|\Omega \backslash D| \\
\quad+\frac{1}{\Theta} \int_{\Omega}\left|\Delta u_{\omega}^{\mathbf{R}}\right|^{2} d x+\frac{1}{\Theta} \Phi_{\mathbf{R}}(\nabla \omega) \int_{\Omega}\left|\nabla u_{\omega}^{\mathbf{R}}\right|^{2} d x .
\end{aligned}
$$

Furthermore, we have

$$
\begin{aligned}
& \left(\frac{1}{2}-\frac{1}{\Theta}\right) \min \{1, a\}\left\|u_{\omega}^{\mathbf{R}}\right\|^{2} \\
& \leq C+a_{1}\left(t_{1}+\frac{\left|t_{1}\right|^{p+1}}{p+1}\right)(\mathbf{R}+2)^{r}|\Omega \backslash D| \\
& \leq C(\mathbf{R}+2)^{r},
\end{aligned}
$$

where $C$ is independence of $b, \mathbf{R}>0$, and $\omega \in \mathbf{H}$. Therefore, $\left\|u_{\omega}^{\mathbf{R}}\right\| \leq v_{2}$, for some $v_{2}:=v_{2}(\mathbf{R})>0$.

Secondly, from $\left(f_{1}\right)$ and $\left(f_{2}\right)$, given $\epsilon>0$, there exists $C_{\epsilon}>0$ such that

$$
f_{\mathbf{R}}\left(x, u_{\omega}^{\mathbf{R}}, \nabla \omega, \Delta \omega\right) \leq \epsilon\left|u_{\omega}^{\mathbf{R}}\right|+C_{\epsilon}(\mathbf{R}+2)^{r}\left|u_{\omega}^{\mathbf{R}}\right|^{p} .
$$

Since $\left\langle J_{\omega}^{\prime \mathbf{R}}\left(u_{\omega}^{\mathbf{R}}\right), u_{\omega}^{\mathbf{R}}\right\rangle=0$, it is easy to obtain that

$$
\min \{1, a\}\left\|u_{\omega}^{\mathbf{R}}\right\|^{2} \leq C_{2} \epsilon\left\|u_{\omega}^{\mathbf{R}}\right\|^{2}+C_{3} C_{\epsilon}\left\|u_{\omega}^{\mathbf{R}}\right\|^{p+1}
$$


for some constants $C_{2}, C_{3} \geq 0$. Therefore, there exists $v_{1}>0$ such that $\left\|u_{\omega}^{\mathbf{R}}\right\| \geq v_{1}$.

Lemma 7 (see [7]). Let $\mathbf{R}$ be fixed, and choose $\omega \in C^{4, \alpha}(\bar{\Omega})$ for $\alpha \in(0,1)$. If $u_{\omega}^{\mathbf{R}} \in \mathbf{X}$ is a weak solution of problem (15), then $u_{\omega}^{\mathbf{R}} \in C^{4, \beta}(\bar{\Omega})$ for some $\beta \in(0,1)$, and $\Delta\left(u_{\omega}^{\mathbf{R}}\right)(x)=0$ if $x \in \partial \Omega$.

Lemma 8. There exist three constants $\eta_{i}>0(i=1,2,3)$, independent of $b, \omega$, and $\mathbf{R}$, such that

$$
\begin{gathered}
\left\|u_{\omega}^{\mathbf{R}}\right\|_{C^{0}} \leq \eta_{1}(\mathbf{R}+2)^{r / 2}, \\
\left\|\nabla u_{\omega}^{\mathbf{R}}\right\|_{C^{0}} \leq \eta_{2}(\mathbf{R}+2)^{r / 2}, \\
\left\|\Delta u_{\omega}^{\mathbf{R}}\right\|_{C^{0}} \leq \eta_{3}(\mathbf{R}+2)^{r / 2} .
\end{gathered}
$$

In addition, there exists $\overline{\mathbf{R}}$ such that

$$
\eta_{i}(\overline{\mathbf{R}}+2)^{r / 2} \leq \overline{\mathbf{R}} \quad(i=1,2,3) .
$$

Proof. From (37) and the proof of Lemma 6, there exists $C>$ 0 , independent of $R>0$ and $\omega \in \mathbf{X}$, such that

$$
\left\|u_{\omega}^{\mathbf{R}}\right\| \leq C(\mathbf{R}+2)^{r / 2} .
$$

Then by Lemma 7 and the Sobolev embedding theorem, the inequalities in the lemma are as follows. In addition, since $r / 2<1$ and $\lim _{s \rightarrow \infty}\left((s+2)^{r / 2} / s\right)=0$, there exists a sufficiently large $\overline{\mathbf{R}}>0$ such that $\theta_{i}(\overline{\mathbf{R}}+2)^{r / 2} \leq \overline{\mathbf{R}}$.

Now let $u_{n}^{\mathrm{R}}(n=1,2, \ldots)$ be the weak solution of the following problem:

$$
\begin{aligned}
\Delta^{2} u_{n}^{\mathbf{R}}-\Phi_{\mathbf{R}}\left(\nabla u_{n-1}^{\mathbf{R}}\right) \Delta u_{n}^{\mathbf{R}} & =f_{\mathbf{R}}\left(x, u_{n}^{\mathbf{R}}, \nabla u_{n-1}^{\mathbf{R}}, \Delta u_{n-1}^{\mathbf{R}}\right), \\
\text { in } \Omega & \\
u_{n}^{\mathbf{R}} & =0, \\
\Delta u_{n}^{\mathbf{R}} & =0,
\end{aligned}
$$

on $\partial \Omega$

with $\omega=u_{n-1}^{\mathbf{R}}$, where $u_{n-1}^{\mathbf{R}}$ was found in Lemma 5 and $\mathbf{R}=$ $\overline{\mathbf{R}}$ obtained in Lemma 8. From Lemmas 6-8, we have $u_{n}^{\mathbf{R}} \in$ $C^{4}(\bar{\Omega})$ satisfying $\left\|u_{n}^{\mathbf{R}}\right\| \geq v_{1}$ and

$$
\left\|u_{n}^{\mathbf{R}}\right\|_{C^{0}},\left\|\nabla u_{n}^{\mathbf{R}}\right\|_{C^{0}},\left\|\Delta u_{n}^{\mathbf{R}}\right\|_{C^{0}} \leq \overline{\mathbf{R}}
$$

Thus

$$
\begin{aligned}
& \Phi_{\mathbf{R}}\left(\nabla u_{n-1}^{\mathbf{R}}\right)=a+b \int_{\Omega}\left|\nabla u_{n-1}^{\mathbf{R}} \varphi_{\mathbf{R}}\left(\nabla u_{n-1}^{\mathbf{R}}\right)\right|^{2} d x \\
& \quad=a+b \int_{\Omega}\left|\nabla u_{n-1}^{\mathbf{R}}\right|^{2} d x, \\
& f_{\mathbf{R}}\left(x, u_{n}^{\mathbf{R}}, \nabla u_{n-1}^{\mathbf{R}}, \Delta u_{n-1}^{\mathbf{R}}\right)=f\left(x, u_{n}^{\mathbf{R}}, \nabla u_{n-1}^{\mathbf{R}}, \Delta u_{n-1}^{\mathbf{R}}\right) .
\end{aligned}
$$

Lemma 9. Assume that $\left(f_{4}\right)$ holds. Let

$$
\begin{aligned}
& \rho_{1}=\inf \left\{\sigma_{1}:\left\|u_{n}^{\mathbf{R}}\right\|_{C^{0}} \leq \sigma_{1}, \forall n \in N^{+}\right\}>0, \\
& \rho_{2}=\inf \left\{\sigma_{2}:\left\|\nabla u_{n}^{\mathbf{R}}\right\|_{C^{0}} \leq \sigma_{2}, \forall n \in N^{+}\right\}>0, \\
& \rho_{3}=\inf \left\{\sigma_{3}:\left\|\Delta u_{n}^{\mathbf{R}}\right\|_{C^{0}} \leq \sigma_{3}, \forall n \in N^{+}\right\}>0 .
\end{aligned}
$$

Then $\left\{u_{n}^{\mathbf{R}}\right\}$ strongly converges in $\mathbf{X}$.

Proof. Let $u_{n+1}^{\mathbf{R}}$ and $u_{n}^{\mathbf{R}}$ be the weak solutions of (43) with $\omega=u_{n}^{\mathbf{R}}$ and $\omega=u_{n-1}^{\mathbf{R}}$, respectively. Then, multiplying (43) by $\left(u_{n+1}^{\mathbf{R}}-u_{n}^{\mathbf{R}}\right)$, we obtain

$$
\begin{gathered}
\min \{1, a\}\left\|u_{n+1}^{\mathbf{R}}-u_{n}^{\mathbf{R}}\right\|^{2} \leq \int_{\Omega}\left[f\left(x, u_{n+1}^{\mathbf{R}}, \nabla u_{n}^{\mathbf{R}}, \Delta u_{n}^{\mathbf{R}}\right)\right. \\
\left.\quad-f\left(x, u_{n}^{\mathbf{R}}, \nabla u_{n-1}^{\mathbf{R}}, \Delta u_{n-1}^{\mathbf{R}}\right)\right]\left(u_{n+1}^{\mathbf{R}}-u_{n}^{\mathbf{R}}\right) d x \\
\quad+b\left(\int_{\Omega}\left|\nabla u_{n}^{\mathbf{R}}\right|^{2} d x\right) \int_{\Omega} \Delta u_{n+1}^{\mathbf{R}}\left(u_{n+1}^{\mathbf{R}}-u_{n}^{\mathbf{R}}\right) d x \\
\quad-b\left(\int_{\Omega}\left|\nabla u_{n-1}^{\mathbf{R}}\right|^{2} d x\right) \int_{\Omega} \Delta u_{n}^{\mathbf{R}}\left(u_{n+1}^{\mathbf{R}}-u_{n}^{\mathbf{R}}\right) d x .
\end{gathered}
$$

Furthermore, by $\left(f_{1}\right),\left(f_{4}\right)$, and the Hölder inequality, we have

$$
\begin{aligned}
\int_{\Omega} & {\left[f\left(x, u_{n+1}^{\mathbf{R}}, \nabla u_{n}^{\mathbf{R}}, \Delta u_{n}^{\mathbf{R}}\right)-f\left(x, u_{n}^{\mathbf{R}}, \nabla u_{n-1}^{\mathbf{R}}, \Delta u_{n-1}^{\mathbf{R}}\right)\right] } \\
& \cdot\left(u_{n+1}^{\mathbf{R}}-u_{n}^{\mathbf{R}}\right) d x \\
& =\int_{\Omega}\left[f\left(x, u_{n+1}^{\mathbf{R}}, \nabla u_{n}^{\mathbf{R}}, \Delta u_{n}^{\mathbf{R}}\right)\right. \\
& \left.-f\left(x, u_{n}^{\mathbf{R}}, \nabla u_{n}^{\mathbf{R}}, \Delta u_{n}^{\mathbf{R}}\right)\right]\left(u_{n+1}^{\mathbf{R}}-u_{n}^{\mathbf{R}}\right) d x \\
& +\int_{\Omega}\left[f\left(x, u_{n}^{\mathbf{R}}, \nabla u_{n}^{\mathbf{R}}, \Delta u_{n}^{\mathbf{R}}\right)\right. \\
& \left.-f\left(x, u_{n}^{\mathbf{R}}, \nabla u_{n-1}^{\mathbf{R}}, \Delta u_{n}^{\mathbf{R}}\right)\right]\left(u_{n+1}^{\mathbf{R}}-u_{n}^{\mathbf{R}}\right) d x \\
& +\int_{\Omega}\left[f\left(x, u_{n}^{\mathbf{R}}, \nabla u_{n-1}^{\mathbf{R}}, \Delta u_{n}^{\mathbf{R}}\right)\right. \\
& \left.-f\left(x, u_{n}^{\mathbf{R}}, \nabla u_{n-1}^{\mathbf{R}}, \Delta u_{n-1}^{\mathbf{R}}\right)\right]\left(u_{n+1}^{\mathbf{R}}-u_{n}^{\mathbf{R}}\right) d x \\
\quad \leq & \tau_{1}^{2} L \rho_{1}\left\|u_{n+1}^{\mathbf{R}}-u_{n}^{\mathbf{R}}\right\|^{2}+\tau_{1} \tau_{2} L_{\rho_{2}}\left\|u_{n}^{\mathbf{R}}-u_{n-1}^{\mathbf{R}}\right\| \\
\quad & \left\|u_{n+1}^{\mathbf{R}}-u_{n}^{\mathbf{R}}\right\|+\tau_{1} \tau_{3} L \rho_{3}\left\|u_{n}^{\mathbf{R}}-u_{n-1}^{\mathbf{R}}\right\| \cdot\left\|u_{n+1}^{\mathbf{R}}-u_{n}^{\mathbf{R}}\right\|, \\
& \quad u_{n}^{\mathbf{R}}\|\cdot\| u_{n}^{\mathbf{R}}-u_{n-1}^{\mathbf{R}} \| \cdot \\
& \left(\int_{\Omega}\left|\nabla u_{n}^{\mathbf{R}}\right|^{2} d x\right) \int_{\Omega}|\Omega|_{n+1}^{1 / 2}\left\|u_{n+1}^{\mathbf{R}}-u_{n}^{\mathbf{R}}\right\|_{L^{2}} \cdot \int_{\Omega}^{\mathbf{R}}\left(\left|\nabla u_{n}^{\mathbf{R}}\right|+\left|\nabla u_{n-1}^{\mathbf{R}}\right|\right) \\
& -b\left(\int_{\Omega}^{\mathbf{R}}\right) d x
\end{aligned}
$$


Hence, by (47) and (48), we get

$$
\begin{aligned}
\min \{1, a\}\left\|u_{n+1}^{\mathbf{R}}-u_{n}^{\mathbf{R}}\right\|^{2} \\
\leq \tau_{1}^{2} L_{\rho_{1}}\left\|u_{n+1}^{\mathbf{R}}-u_{n}^{\mathbf{R}}\right\|^{2}+\tau_{1} \tau_{2} L_{\rho_{2}}\left\|u_{n}^{\mathbf{R}}-u_{n-1}^{\mathbf{R}}\right\| \\
\cdot\left\|u_{n+1}^{\mathbf{R}}-u_{n}^{\mathbf{R}}\right\|+\tau_{1} \tau_{3} L_{\rho_{3}}\left\|u_{n}^{\mathbf{R}}-u_{n-1}^{\mathbf{R}}\right\| \\
\cdot\left\|u_{n+1}^{\mathbf{R}}-u_{n}^{\mathbf{R}}\right\|+2 b \rho_{2} \rho_{3} \tau_{1} \tau_{2}|\Omega|\left\|u_{n+1}^{\mathbf{R}}-u_{n}^{\mathbf{R}}\right\| \\
\cdot\left\|u_{n}^{\mathbf{R}}-u_{n-1}^{\mathbf{R}}\right\| ;
\end{aligned}
$$

namely,

$$
\begin{aligned}
& \left\|u_{n+1}^{\mathbf{R}}-u_{n}^{\mathbf{R}}\right\| \\
& \quad \leq \frac{\tau_{1} \tau_{2} L_{\rho_{2}}+\tau_{1} \tau_{3} L_{\rho_{3}}+2 b \rho_{2} \rho_{3} \tau_{1} \tau_{2}|\Omega|}{\min \{1, a\}-L_{\rho_{1}} \tau_{1}^{2}}\left\|u_{n}^{\mathbf{R}}-u_{n-1}^{\mathbf{R}}\right\| .
\end{aligned}
$$

Now, choosing $b^{*}=\left(\min \{1, a\}-L_{\rho_{1}} \tau_{1}^{2}-L_{\rho_{2}} \tau_{1} \tau_{2}-L_{\rho_{3}} \tau_{1} \tau_{3}\right) /$ $2 \rho_{2} \rho_{3} \tau_{1} \tau_{2}|\Omega|$, then

$$
\frac{\tau_{1} \tau_{2} L_{\rho_{2}}+\tau_{1} \tau_{3} L_{\rho_{3}}+2 b \rho_{2} \rho_{3} \tau_{1} \tau_{2}|\Omega|}{\min \{1, a\}-L_{\rho_{1}} \tau_{1}^{2}}<1,
$$

$$
\text { for } 0<b<b^{*} \text {. }
$$

Therefore, $\left\{u_{n}^{\mathbf{R}}\right\}$ converges strongly in $\mathbf{X}$.

Proof of Theorem 2. Firstly, from Lemma 6, we get $\left\|u_{n}^{\mathbf{R}}\right\| \geq$ $\nu_{1}>0$, and $\left\|u_{n}^{\mathbf{R}}\right\|_{C^{0}},\left\|\nabla u_{n}^{\mathbf{R}}\right\|_{C^{0}},\left\|\Delta u_{n}^{\mathbf{R}}\right\|_{C^{0}}$ are uniformly bounded. Secondly, set $v_{n}=\Delta u_{n}^{\mathbf{R}}$; then

$$
\begin{aligned}
\Delta v_{n}= & h(x) \\
= & f\left(x, u_{n}^{\mathbf{R}}, \nabla u_{n-1}^{\mathbf{R}}, \Delta u_{n-1}^{\mathbf{R}}\right) \\
& +\left(a+b \int_{\Omega}\left|\nabla u_{n-1}^{\mathbf{R}}\right|^{2} d x\right) \Delta u_{n}^{\mathbf{R}} .
\end{aligned}
$$

Since $\|h\|_{C^{\beta}} \leq C$, for some positive constant $C$, by the Schauder theorem, there exists a constant $C_{0}>0$ such that $\left\|v_{n}\right\|_{C^{2, \beta}} \leq C_{0}$; that is, $\left\|u_{n}^{\mathbf{R}}\right\|_{C^{4, \beta}} \leq C_{0}$. Furthermore, by the Arzela-Ascoli theorem and Lemma 9 , the sequence $\left\{u_{n}^{\mathbf{R}}\right\}$ satisfies

$$
\frac{\partial^{j}}{\partial x_{i}^{j}} u_{n}^{\mathbf{R}}(x) \longrightarrow \frac{\partial^{j}}{\partial x_{i}^{j}} u^{\mathbf{R}}(x), \quad \text { as } n \longrightarrow \infty
$$

uniformly in $\bar{\Omega}$ for $j=0,1,2,3,4$ and $i=1, \ldots, N$. Finally, passing to the limit in (43), we obtain that $u^{\mathbf{R}}(x)$ is a classical solution of (1).

Example 10. Consider the following problem:

$$
\Delta^{2} u-\left(a+b \int_{\Omega}|\nabla u|^{2} d x\right) \Delta u=f(x, u, \nabla u, \Delta u),
$$

in $\Omega$, $u=0$

$\Delta u=0$,

on $\partial \Omega$,

where $f\left(x, t, \xi_{1}, \xi_{2}\right)=\alpha(x)|t| t\left(1+\left|\xi_{1}\right|\right)^{1 / 2}\left(1+\left|\xi_{2}\right|\right)^{3 / 4}+\beta(x) t^{3}$ and $\alpha(x)$ and $\beta(x)$ are positive and continuous functions. It is easy to verify that $f\left(x, t, \xi_{1}, \xi_{2}\right)$ satisfies all the conditions of $\left(f_{1}\right)-\left(f_{4}\right)$.

\section{Conclusion}

The paper considers a class of fourth-order elliptic equations of Kirchhoff type with dependence on the gradient and Laplacian. The existence of a nontrivial solution of (1) is established when we choose appropriate $b^{*}$ such that $0<$ $b<b^{*}$. The paper generalized the conclusions in $[7,14]$ and weakened the condition in [7]. In the following research work, we will also consider problem (1), but we just truncate the right side of the equation, and the left of the equation remains the same.

\section{Conflicts of Interest}

The authors declare that they have no conflicts of interest.

\section{Authors' Contributions}

All the authors contributed equally and significantly to writing this article. All the authors read and approved the final manuscript.

\section{Acknowledgments}

Yunhai Wang was supported by the projects of Guizhou Provincial Science and Technology Fund (QKH-JICHU [2017], Grant no. 1408) and the doctoral starting up foundation of Guizhou Institute of Technology (Grant no. XJGC20150408). Fanglei Wang was supported by NNSF of China (no. 11501165) and the Fundamental Research Funds for the Central Universities (2015B19414).

\section{References}

[1] P. J. McKenna and W. Walter, "Travelling waves in a suspension bridge," SIAM Journal on Applied Mathematics, vol. 50, no. 3, pp. 703-715, 1990 .

[2] A. M. Micheletti and A. Pistoia, "Multiplicity results for a fourth-order semilinear elliptic problem," Nonlinear Analysis. Theory, Methods \& Applications. An International Multidisciplinary Journal, vol. 31, no. 7, pp. 895-908, 1998.

[3] Y. An and R. Liu, "Existence of nontrivial solutions of an asymptotically linear fourth-order elliptic equation," Nonlinear Analysis. Theory, Methods \& Applications. An International Multidisciplinary Journal, vol. 68, no. 11, pp. 3325-3331, 2008.

[4] S. Hu and L. Wang, "Existence of nontrivial solutions for fourthorder asymptotically linear elliptic equations," Nonlinear Analysis. Theory, Methods \& Applications. An International Multidisciplinary Journal, vol. 94, pp. 120-132, 2014. 
[5] Y.-M. Wang, "On fourth-order elliptic boundary value problems with nonmonotone nonlinear function," Journal of Mathematical Analysis and Applications, vol. 307, no. 1, pp. 1-11, 2005.

[6] G. Xu and J. Zhang, "Existence results for some fourthorder nonlinear elliptic problems of local superlinearity and sublinearity," Journal of Mathematical Analysis and Applications, vol. 281, no. 2, pp. 633-640, 2003.

[7] P. C. Carriao, L. F. Faria, and O. m. Miyagaki, "A biharmonic elliptic problem with dependence on the gradient and the Laplacian," Electronic Journal of Differential Equations, vol. 93, pp. 1-12, 2009.

[8] D. De Figueiredo, M. Girardi, and M. Matzeu, "Semilinear elliptic equations with dependence on the gradient via mountain-pass techniques," Differential and Integral Equations. An International Journal for Theory \& Applications, vol. 17, no. 1-2, pp. 119-126, 2004.

[9] M. Girardi and M. Matzeu, "Positive and negative solutions of a quasi-linear elliptic equation by a mountain pass method and truncature techniques," Nonlinear Analysis. Theory, Methods \& Applications. An International Multidisciplinary Journal, vol. 59, no. 1-2, pp. 199-210, 2004.

[10] F. J. Correea and G. M. Figueiredo, "On an elliptic equation of $p$-Kirchhoff type via variational methods," Bulletin of the Australian Mathematical Society, vol. 74, no. 2, pp. 263-277, 2006.

[11] T. F. Ma, "Positive solutions for a nonlocal fourth order equation of Kirchhoff type," Discrete and Continuous Dynamical Systems - Series A, pp. 694-703, 2007.

[12] T. F. Ma, "Positive solutions for a nonlinear Kirchhoff type beam equation," Applied Mathematics Letters, vol. 18, no. 4, pp. 479482, 2005.

[13] T. F. Ma and A. L. Martinez, "Positive solutions for a fourth order equation with nonlinear boundary conditions," Mathematics and Computers in Simulation, vol. 80, no. 11, pp. 21772184, 2010.

[14] F. Wang, M. Avci, and Y. An, "Existence of solutions for fourth order elliptic equations of Kirchhoff type," Journal of Mathematical Analysis and Applications, vol. 409, no. 1, pp. 140-146, 2014.

[15] M. Ferrara, S. Khademloo, and S. Heidarkhani, "Multiplicity results for perturbed fourth-order Kirchhoff type elliptic problems," Applied Mathematics and Computation, vol. 234, pp. 316325, 2014.

[16] S. Heidarkhani, S. Khademloo, and A. Solimaninia, "Multiple solutions for a perturbed fourth-order Kirchhoff type elliptic problem," Portugaliae Mathematica. A Journal of the Portuguese Mathematical Society, vol. 71, no. 1, pp. 39-61, 2014.

[17] S. Heidarkhani, M. Ferrara, and S. Khademloo, "Nontrivial solutions for one-dimensional fourth-order Kirchhoff-type equations," Mediterranean Journal of Mathematics, vol. 13, no. 1, pp. 217-236, 2016.

[18] A. Nazari, G. A. Afrouzi, and S. Heidarkhani, "Infinitely many solutions for perturbed fourth-order Kirchhoff-type problems," Dynamic Systems and Applications, vol. 25, no. 1-2, pp. 237-251, 2016.

[19] M. R. Heidari Tavani, G. A. Afrouzi, and S. Heidarkhani, "Multiplicity results for perturbed fourth-order Kirchhoff-type problems," Opuscula Mathematica, vol. 37, no. 5, pp. 755-772, 2017.

[20] S. Heidarkhani, G. A. Afrouzi, M. Ferrara, and S. Moradi, "Variational approaches to impulsive elastic beam equations of Kirchhoff type," Complex Variables and Elliptic Equations. An International Journal, vol. 61, no. 7, pp. 931-968, 2016. 


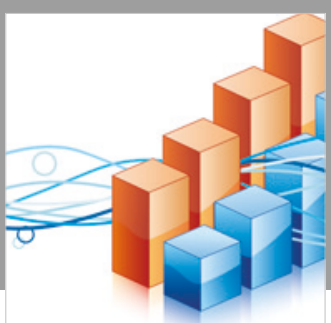

Advances in

Operations Research

\section{-n-m}
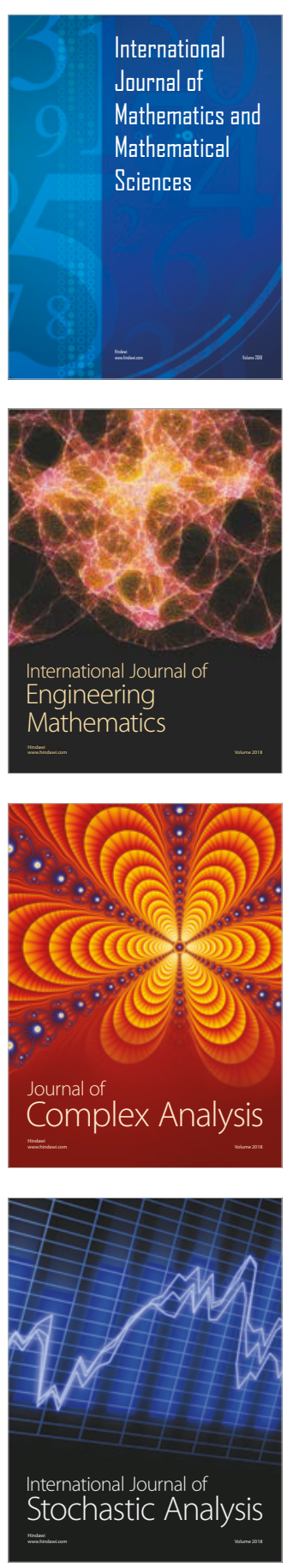
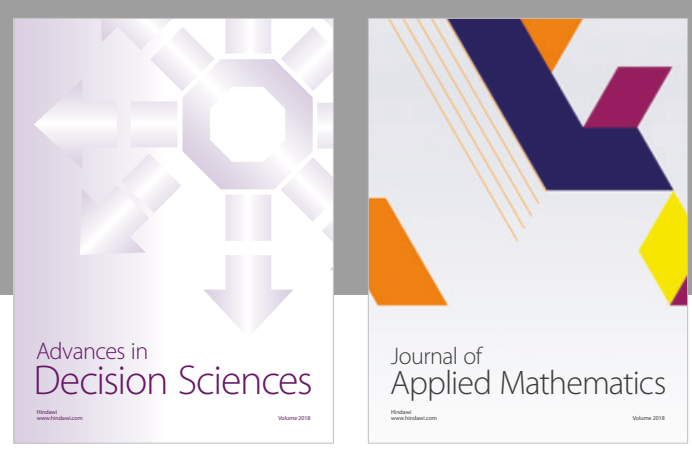

Journal of

Applied Mathematics
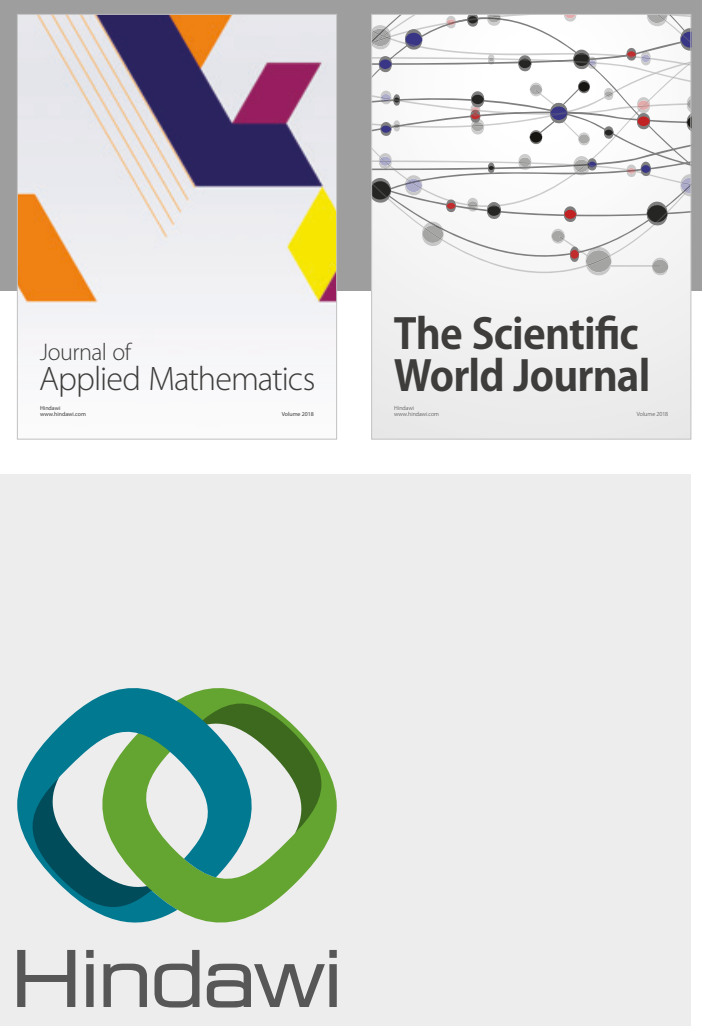

Submit your manuscripts at

www.hindawi.com

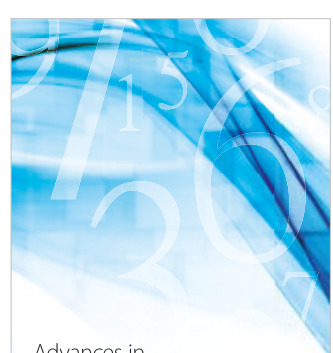

Advances in
Numerical Analysis
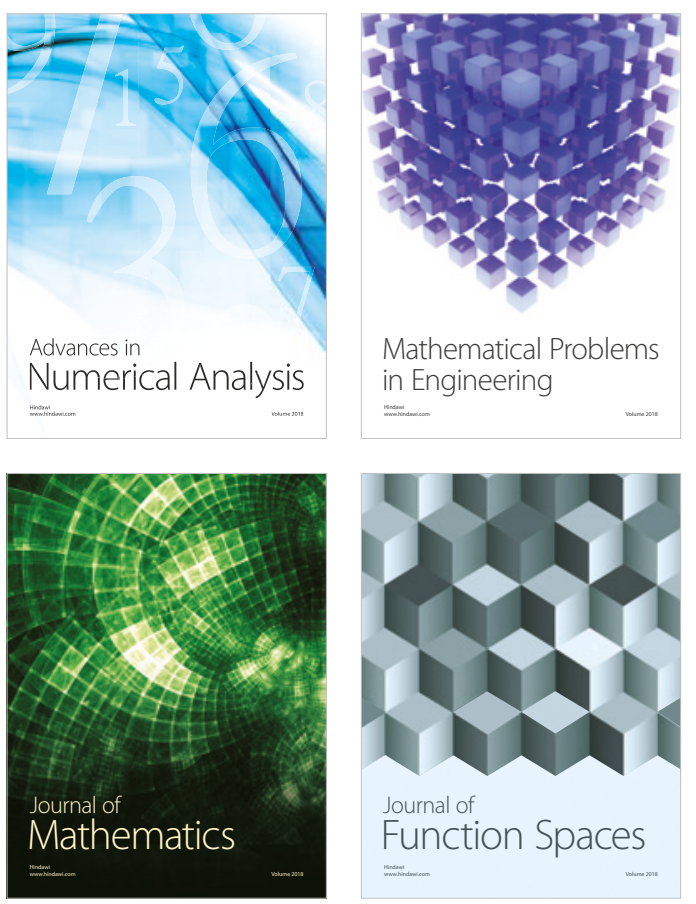

Mathematical Problems in Engineering

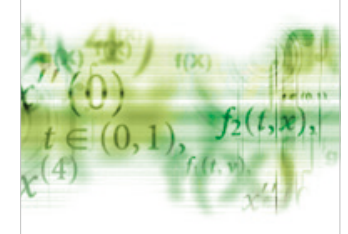

International Journal of

Differential Equations

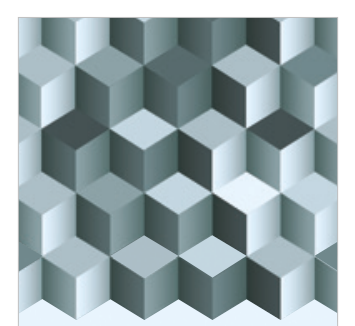

Journal of

Function Spaces

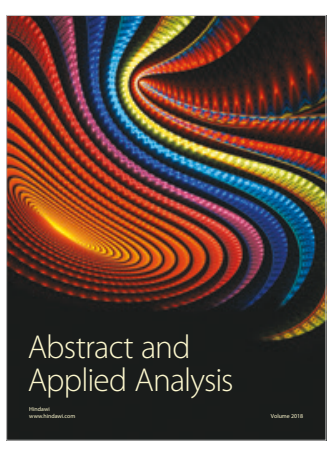

The Scientific

World Journal

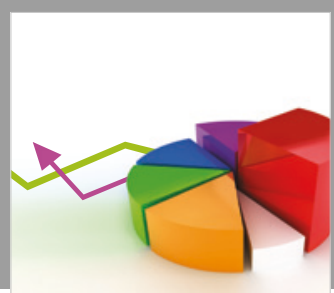

Journal of

Probability and Statistics
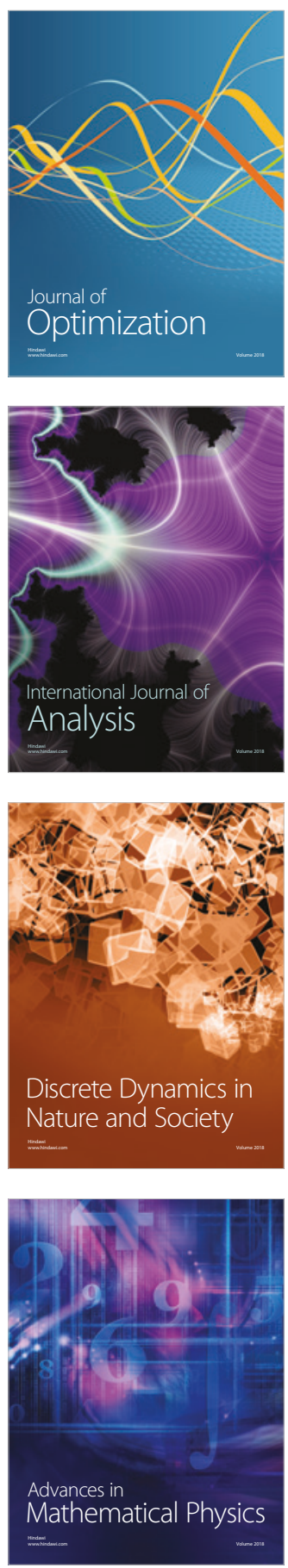